\title{
The significance of the determination of lymphocytes with clinical manifestation of ophthalmic zoster sine herpete
}

This article was published in the following Dove Press journal:

Clinical Ophthalmology

15 July 2010

Number of times this article has been viewed

Tatsuya Marumoto'

Yoshimune Hiratsuka ${ }^{2,3}$

Akira Murakami²

'Marumoto Eye Clinic, Yokohama, Japan; ${ }^{2}$ Department of Ophthalmology, Juntendo University School of Medicine, Tokyo, Japan; ${ }^{3}$ Department of Management Sciences, National Institute of Public Health, Wako, Japan
Objective: To examine the significance of VZV-IgG (IgG) enzyme immunoassay (EIA) and the determination of peripheral blood lymphocyte counts in the diagnosis of ophthalmic zoster sine herpete (ophthalmic ZSH).

Method: Clinical case-control study with a sample of 65 patients with ophthalmic ZSH (16 males and 49 females; mean age $56 \pm 18$ years) in whom pressure elicited pain in tender points of Valleix (tender points of sites at which the trigeminal nerve appears subcutaneously). A total of 41 healthy controls ( 17 males and 24 females, mean age $48 \pm 21$ years) were also recruited. In each group, IgG and lymphocyte count were determined upon receiving the consent of patients. Multiple logistic regression analysis was used to evaluate the factors associated with diagnosis of ophthalmic ZSH. Odds ratios and 95\% confidence intervals (CI) were determined for each variable.

Results: Lymphocyte count in the patients with ophthalmic ZSH was significantly low, though there were no significant differences in age, gender, and IgG between the two groups. Multiple logistic regression analysis revealed that lymphocytes pose a significant prognostic factor, and in case of 1,800 lymphocytes/ $\mu 1$ or more, the odds ratio to less than 1,800 lymphocytes $/ \mu 1$ was 0.29 (95\% CI: 0.12-0.72).

Conclusion: Patients with orbital pain should be suspected as having ophthalmic ZSH as this allows for the determination of lymphocyte counts after tender points were confirmed. The swift diagnosis of ophthalmic ZSH and the administration of antiviral drugs from an early stage improve the subjective symptoms of the patient and is regarded as necessary to reduce the risk of severe complications.

Keywords: ophthalmic ZSH, lymphocytes count, VZV-IgG, tender points of Valleix, orbital pain, antiviral drugs

Zoster sine herpete (ZSH) has been defined as pain in a dermatomal distribution without the rash of cutaneous zoster. ${ }^{1}$ Ophthalmic ZSH, or ZSH involvement of the ophthalmic division of the trigeminal nerve, results from the reactivation of chickenpox and varicella zoster virus (VZV), which are dormant in the trigeminal ganglion. It causes various symptoms such as trigeminal neuralgia, headache, and malaise. ${ }^{2}$ Ophthalmic ZSH is characterized by spontaneous pain in the area of the first branch of the trigeminal nerve, which is considered to be an important - and the only subjective - symptom. ${ }^{3}$ Its diagnosis is difficult as blisters do not develop and there is no clue to its diagnosis other than orbital pain. ${ }^{4}$ For this reason, although a reliable diagnosis is difficult, early diagnosis is important as ZSH has also been reported to cause severe uveitis, ${ }^{5,6}$ acute retinal necrosis syndrome, ${ }^{7,8}$ and neuroparalysis ${ }^{9}$ if left untreated. 
So far, elevated anti-VZV antibody titers have been reported as a useful finding for the diagnosis of ophthalmic herpes zoster; ${ }^{10}$ however, there are no reports concerning ophthalmic ZSH.

Herpes zoster is particularly common among patients with lymphoproliferative malignancies. ${ }^{11}$ In 1980, Miller found that lymphocyte proliferation to varicella-zoster antigen was lower in older asymptomatic individuals than in normal young controls. ${ }^{12}$ The precise roles of humoral and cellular immunity in protection against herpes zoster virus infection are controversial. However, cell-mediated immunity seems to be more important than humoral immunity, probably due to the spread of virus within the body which is exclusively via the intracellular route.

We considered that the lymphocyte count may trigger of onset process of ophthalmic ZSH, so our purpose in this study was to examine the significance of the determination of enzyme immunoassay (EIA), VZV-IgG (immunoglobulin G) levels (IgG), and peripheral blood lymphocyte counts (lymphocyte count) in the early diagnosis of ophthalmic ZSH.

\section{Method}

Only people who provided informed consent were eligible to participate in this study. Those involved were patients who visited the Marumoto Eye Clinic (Yokahama) between August 2008 and February 2009. Our study design features a clinical case-control study. There were 65 patients with ophthalmic ZSH (16 males and 49 females; mean age $56.3 \pm 18.4$ years) in whom pressure elicited pain in tender points of Valleix ${ }^{13}$ (tender points of sites at which the trigeminal nerve appears subcutaneously). By pushing the patient's upper eyelid with a forefinger over the eyeball, it becomes easier to elicit the pain of the tender points. Ophthalmic ZSH was suspected upon medical examination by the interviewers, and for treatment internal use of valacyclovir alone was administered and therapeutic diagnosis was performed. For discriminating from episcleritis or scleritis, all cases had been prescribed only valacyclovir and no topical steroids. Controls comprised 41 participants (17 males and 24 females, mean age $48.2 \pm 20.9$ years) in whom pressure did not elicit pain in tender points of Valleix. Controls had visited the clinic during the same period as patients and received reports of cataract and refractive error. Controls were free from VZV disease and we gained consent to carry out the study with all participants. In each group, IgG and lymphocyte count were determined (outside ordered to Health Sciences Research Institute) upon receiving the consent of the patients.
Pearson's Chi-square tests and Mann-Whitney tests were conducted to explore the differences between participants with ophthalmic ZSH and the control group in terms of age, sex, IgG levels, and lymphocyte count. Multiple logistic regression analysis was used to evaluate the factors associate with the diagnosis of ophthalmic ZSH. First, all variables of age, gender, IgG levels, and lymphocyte count were analyzed in a univariate model. Then all variables were included in a multivariate model. Statistical significance was considered when $P<0.05$ was reached. The role of each variable is expressed in odds ratio (OR) and the related $95 \%$ confidence intervals (CI). Computerized analysis was performed using STATA software version 8 (Statacorp., College Station, Texas, US).

\section{Results}

A total of 65 patients with ophthalmic ZSH and 41 controls were enrolled in the present study. Table 1 presents the symptoms and conditions of ophthalmic ZSH cases. In the ophthalmic ZSH group, in which participants experienced tenderness, $100 \%$ of the patients had an injection in the eye and almost $60 \%$ of the patients experienced lacrimation, headaches, and a stiff neck. After a course of valacyclovir, in all cases spontaneous pain and pain felt upon exerting pressure disappeared within 1 week, improving the subjective symptoms of the patients.

Table I Symptoms and conditions of ZSH cases

\begin{tabular}{ll}
\hline Symptoms & $\begin{array}{l}\text { Cases }(\mathbf{N}=\mathbf{6 5}) \\
\text { no. of patients (\%) }\end{array}$ \\
\hline Tenderness & $100(65)$ \\
Orbital pain & $32(21)$ \\
Injection & $100(65)$ \\
Sandy sensation in eyes & $48(31)$ \\
Lacrimation & $63(41)$ \\
Dry eye & $37(24)$ \\
Headache & $58(38)$ \\
Stiff neck & $62(40)$ \\
Lassitude & $49(32)$ \\
Dizziness & $32(21)$ \\
Conditions & no. of patients (\%) \\
\hline Overwork & $75(49)$ \\
Stress & $60(39)$ \\
The common cold & $58(38)$ \\
Lack of sleep & $68(44)$ \\
Irregular hours & $23(15)$ \\
History of VZV & $32(21)$ \\
Number of recurrence & $23(15)$ \\
\hline & \\
\hline & \\
\hline
\end{tabular}


Details regarding age, gender, $\operatorname{IgG}$, and lymphocyte count of the patients with ophthalmic ZSH and the control group are shown in Table 2. In both groups there were no significant differences in age, gender, and IgG. On the other hand, mean lymphocyte count was $1,765 \pm 550$ and 2,276 \pm 715 lymphocytes/ $\mu 1$ in the patients with ophthalmic ZSH and the control group, respectively, and was thus significantly lower in patients with ophthalmic ZSH ( $P=0.0003$, Mann-Whitney test).

Furthermore, multiple logistic regression analysis using the absence of ophthalmic ZSH as dependent variable, and age, gender, IgG levels, and lymphocyte count as prognostic factors, was performed. Prognostic factors were classified as follows, age: per 1 year of age, gender: male/female, IgG levels: less than 16 and 16 or more, lymphocyte count: less than 1,800 lymphocytes $/ \mu 1$ and 1,800 lymphocytes $/ \mu 1$ or more. As a result of univariate analysis, age (OR, 1.02; 95\% CI, 1.00-1.04) and lymphocyte count (OR, 0.28; $95 \% \mathrm{CI}, 0.12-0.66$ ) were found to be significant prognostic factors. Multivariate analysis adjusted for age, gender, and IgG levels revealed that only lymphocyte count was a significant prognostic factor, and in the case of 1,800 lymphocytes/ $\mu 1$ and more, OR of less than 1,800 lymphocytes/ $\mu 1$ was 0.29 (95\% CI, 0.12-0.72; Table 3).

\section{Discussion}

Ophthalmic ZSH was first described by Ross in $1949 .{ }^{14}$ In 1958 Lewis reported 13 cases of ZSH among 120 cases of herpes zoster. ${ }^{3} \mathrm{VZV}$ is dormant in the trigeminal ganglion of almost $100 \%$ of the Japanese population and is reactivated along with a decrease in the resistance power of the body, therefore causing the development of trigeminal neuralgia and shingles in the trigeminal nerve area. ${ }^{15}$ Although various symptoms are produced as a result of the reactivation of this VZV, ophthalmic ZSH is a disease in which no shingles appear, and except for secondary trigeminal neuralgia caused by tumors of the trigeminal nerve, intracranial aneurysm,

Table 2 Comparison of all prognostic factors

\begin{tabular}{|l|c|c|c|}
\hline & $\begin{array}{c}\text { ZSH group } \\
n=65 \\
(m e a n \pm S D)\end{array}$ & $\begin{array}{c}\text { Control group } \\
n=41 \\
(m e a n \pm S D)\end{array}$ & \\
\hline Age (years) & $56.3 \pm 18.4$ & $48.2 \pm 20.9$ & $P=0.0644^{*}$ \\
\hline Gender (M/F Ratio) & $16 / 49$ (cases) & $17 / 24$ (cases) & $P=0.0681^{* *}$ \\
\hline VZV-IgG & $19.8 \pm 17.1$ & $15.9 \pm 11.3$ & $P=0.4023^{*}$ \\
\hline Lymphocyte count & $1,765 \pm 550$ & $2,276 \pm 715$ & $P=0.0003^{*}$ \\
\hline
\end{tabular}

Notes: *Mann-Whitney test, ${ }^{* *}$ Chi-Square test or paranasal sinus disease, pain is expected to completely disappear within weeks. ${ }^{16}$

It is speculated that the various symptoms found in the experimental group were produced as a result of reactivation of VZV. For example, a sandy sensation and lacrimation may be caused by hyperaesthesia of the trigeminal nerve, and these symptoms may be ignored as indefinite complaints. In this study, the ophthalmic ZSH group complained of general discomfort, such as headaches along the temples, stiff necks and shoulders, dizziness, and lassitude, which may have resulted from inflammation of the trigeminal nerve. These results suggested that the indefinite complaints may be able to be eliminated in response to antiviral therapy. In all cases, treatment involved internal use of valacyclovir alone and no steroids for topical or internal use were administered. Through systemic use of valacyclovir from an early stage at a low dose (daily dose of $1500 \mathrm{mg}, 3$ times a day for 5 days), in all cases spontaneous pain and pain felt upon exerting pressure disappeared within 1 week, improving the subjective symptoms of the patients.

It is known that hemicrania is accompanied by allodynia, ${ }^{17}$ making patients feel a stinging pain from their hair and skin just by wind that is blowing against their forehead or around their eyes. ${ }^{18}$ Shimizu reported a 4.3 times higher rise in the development of allodynia for a group of hemicrania patients with high anti-VZV antibody titers compared to a group of hemicrania patients with low antiVZV antibody titers (cut-off value of 4 times or more for CF).${ }^{19}$ Furthermore, these symptoms have been reported to improve through internal use of valacyclovir. These results suggest that VZV is involved in hemicrania accompanied by allodynia. In this study, together with allodynia, which produces spontaneous pain as subjective findings, we focused on tender points. At these sites, which are called tender points of Valleix, the trigeminal nerve appears subcutaneously. These sites are hypersensitive in case of trigeminal neuralgia and have been used for decades in the diagnosis of neuralgia. ${ }^{13}$ For the diagnosis of ophthalmic ZSH, the only pain that can be identified by hand diagnosis upon exerting pressure is considered significant for determining the hypersensitivity of the trigeminal nerve. ${ }^{13}$ The pain felt upon exerting pressure also is present when no spontaneous pain shows up and tender points could easily be identified. It has been speculated that although reactivated VZV does not cause a rash or headache, it lowers the pain threshold of trigeminal neurons, and thus causes excitability. All of the patients with ophthalmic ZSH felt pain in the tender points of both eyes upon exerting pressure; however, the pain in 
Table 3 Logistic regression analysis

\begin{tabular}{|c|c|c|c|c|c|}
\hline \multirow[t]{2}{*}{ ZSH } & & \multicolumn{2}{|c|}{ Univariate analysis } & \multicolumn{2}{|c|}{ Multivariate analysis } \\
\hline & & Odds ratio & $\begin{array}{c}95 \% \\
\text { confidence } \\
\text { interval }\end{array}$ & $\begin{array}{l}\text { Odds ratio } \\
\text { (adjusted for } \\
\text { other factors) }\end{array}$ & $\begin{array}{c}95 \% \\
\text { confidence } \\
\text { interval }\end{array}$ \\
\hline Age & $\begin{array}{l}\text { Per lyear } \\
\text { of age }\end{array}$ & 1.02 & $1.00-1.04$ & 1.02 & $1.00-1.04$ \\
\hline \multirow[t]{2}{*}{ Gender } & Female & I & & I & \\
\hline & Male & 0.47 & $0.29-1.07$ & 0.43 & $0.17-1.06$ \\
\hline \multirow[t]{2}{*}{ VZV-lgG } & $<16$ & I & & I & \\
\hline & $16 \leq$ & 1.60 & $0.7 I-3.60$ & 1.02 & $0.56-3.27$ \\
\hline \multirow[t]{2}{*}{ Lymphocyte count } & $<1,800$ & I & & I & \\
\hline & $1,800 \leq$ & 0.28 & $0.12-0.66$ & 0.29 & $0.12-0.72$ \\
\hline
\end{tabular}

one eye was stronger than in the other. Depending on the case, there were differences in the level of pain felt upon exerting pressure; in many cases the pain that was felt upon exerting pressure on the superior eyelid over the eyeball on the more painful side was so strong that the patient tried to move away. VZV-related disorders commonly occur in one eye; however, in case of ophthalmic ZSH the development of symptoms in both eyes has been reported. ${ }^{20}$ The reason why pain felt upon exerting pressure was confirmed for both eyes is not because the inflammation of the trigeminal nerve of one eye spread to the other side, but because when the pain in the one eye is anterogradely passed on to the brain and the pain information is recognized, this information is antidromically transmitted to the opposite trigeminal ganglion. ${ }^{19}$ Furthermore, although the level of injection of conjunctiva was not necessarily reciprocated with the intensity of the pain felt upon exerting pressure, congestion characterized by overswelling of only a number of blood vessels of the bulbar conjunctiva of two cases was observed.

The virological investigation of VZV includes virus isolation, virus antigen (or DNA) detection, and the determination of specific antibody titers. ${ }^{14}$ As for the isolation of the virus, liquid from herpes blisters is used (although this method cannot be used for ophthalmic $\mathrm{ZSH}$ in which no rash develops). ${ }^{10}$ Additionally, for the detection of virus antigens, VZV-DNA in aqueous humor of the anterior eye chamber is quantitatively determined (real time PCR); however, this requires the collection of aqueous humor of the anterior eye chamber, and thus is unrealistic in general clinical practice. ${ }^{6}$ On the other hand, the determination of a VZV-specific serum antibody titer is done by taking blood samples. Methods used for the determination of specific antibody titers are complement-fixation reaction $(\mathrm{CF})$, EIA, neutralization, fluorescent antibody test, and more. Currently, CF and EIA are often used in clinical practice. CF is superior with regard to simplicity and swiftness of the examination method, and also has the advantage that it can be done at a moderate price (about one-third that of EIA). However, both sensitivity and specificity are lower than in EIA $^{21}$ and as often as cross-reactivity with the herpes simplex virus occurs, data must be handled with care. ${ }^{22}$ Furthermore, for a reliable serological diagnosis, CF levels of paired serum (an interval of at least two weeks) from the acute and the recovery phase must be compared and a rise in two or more stages must be confirmed. It is difficult to compare pair serum in day-to-day ophthalmic practice and in fact this is not often done. On the other hand, by EIA, single sera can be diagnosed and it is said that VZV infections can be detected if the assay is performed at least within 2 years after the onset of the infection, while in case of CF, sera are said to become diagnostically useless 6 months or more after onset of the infection. ${ }^{21}$ For these reasons, in the present study, we determined virus antibody titers by EIA. However, as a result no significant differences in IgG levels were observed between the patients with ophthalmic ZSH and the control group (Table 2). Furthermore, Yamakawa ${ }^{9}$ set the positive cut-off standard, which is defined as an abnormally high IgG 
value at an EIA value of 50; however, in our study, only in 4 of the 65 participants of the patients with ophthalmic ZSH was an $\mathrm{IgG}$ of 50 or more observed, while the mean value was 18 . This result let us assume that the IgG value provides no helpful information for the early diagnosis of ophthalmic ZSH. The reason is that in the case of ophthalmic ZSH no rash develops, and ophthalmic ZSH is believed to be an inflammation that is confined to the trigeminal and occipital nerve. In the stage of the early diagnosis of ophthalmic ZSH through identifying pain that is felt upon exerting pressure, no anti-VZV antibody, including immunoglobulin (humoural immunity), has yet elicited a strong response. On the other hand, high IgG levels (maximum value 45.9) were also observed in the healthy control cases. Generally, IgG is said to show the history of all VZV reactivations during the last 2 years, ${ }^{22}$ and thus do not necessarily capture the present VZV-reactivation state.

Regarding lymphocyte counts, univariate analysis also revealed significant differences between the two groups. Furthermore, as a result of multivariate analysis in which the patients with ophthalmic ZSH were separated into two groups with the medium value of the normal lymphocyte count of 1,800 lymphocytes/ $\mu 1$ as limit, it was revealed that lymphocyte counts had a relevance three times higher in the less than 1,800 lymphocytes/ $\mu$ l group, compared to the 1,800 lymphocytes/ $\mu 1$ or more group. Upon medical examination by the interviewers of all of the patients with ophthalmic ZSH, one or more of the following complaints were reported: stress, lack of sleep, coldness in one's hands and feet, or irregular lifestyle. In a state in which the sympathetic nerves are superior as described above, lymphocyte counts in serum decrease. This is because an increase in the lymphocyte count in serum is controlled by the parasympathetic nerves and in a state in which the sympathetic nerves are superior, the lymphocyte count decreases. ${ }^{23,24}$ There is a possibility that a decrease in the lymphocyte count triggered VZV reactivation and the onset of ophthalmic ZSH.

In conclusion, we believe that patients with orbital pain should be tested for the presence of ophthalmic $\mathrm{ZSH}$, and that it is useful to determine lymphocyte counts after tender points are confirmed. The swift diagnosis of ophthalmic $\mathrm{ZSH}$ and the administration of antiviral drugs from an early stage improve the subjective symptoms of the patient and is regarded necessary to reduce the risk of severe complications.

\section{Acknowledgment}

The authors thank Dr Kouichi Ono of the Juntendo University School of Medicine, Tokyo, Japan.

\section{Disclosure}

The authors report no conflicts of interest in this work.

\section{References}

1. Elliott KJ. Other neurological complications of herpes zoster and their management. Ann Neurol. 1994;35:57-61.

2. Liesegang TJ. Herpes zoster ophthalmicus. Ophthalmol. 2007;10: $3-12$.

3. Lewis GW. Zoster sine herpete. Br Med J. 1958;16:418-421.

4. Kanski JJ. Viral uveitis. In: Butterworth H, editor. Clinical Ophthalmology. Philadelphia, Pennsylvania: Elsevier Science Limited. 2003; 271-308.

5. Kido S, Sugita S, Horie S, et al. Association of varicella zoster virus load in the aqueous humor with clinical manifestations of anterior uveitis in herpes zoster ophthalmicus and zoster sine herpete. Br J Ophthalmol. 2008;92:505-508.

6. Kezuka T, Sakai J, Minoda H, et al. A relationship between varicellazoster virus-specific delayed hypersensitivity and varicella-zoster virusinduced anterior uveitis. Arch Ophthalmol. 2002;120:1183-1188.

7. Culbertson WW, Blumenkranz MS, Pepose JS, et al. Varicella zoster virus is a cause of the acute retinal necrosis syndrome. Ophthalmol. 1986;93:559-569.

8. Kezuka T, Sakai J, Usui N, et al. Evidence for antigen-specific immune deviation in patients with acute retinal necrosis. Arch Ophthalmol. 2001;119:1044-1049.

9. Yamakawa K, Hamada M, Takeda T. Assessment of anti-VZV IgG antibodies in patients with facial palsy. Comparison between CF, FA, and EIA. Facial N Res Jpn. 2004;24:53-56.

10. Sasaki K, Iida K, Nejima R. Is serological diagnosis (IgG by EIA method) useful in diagnosing persistent keratitis following herpes zoster ophthalmicus? Eye. 2007;24:502-504.

11. Goffinett DR, Glatstein EJ, Merigan TC. Herpes zoster-varicella infections and lymphoma. Ann Intern Med. 1972;76:235-240.

12. Miller A. Selective decline in cellular immune response to varicellazoster in the elderly. Neurol. 1980;30:582-587.

13. Valleix FL. Traite des neuralgies ou affections douloureuses des nerfs, 1st ed. Paris J B Bailliere 8vo. Contemp. leather backed bds. $1841 ; 2-719$.

14. Ross JM. Herpes zoster ophthalmicus sine eruptione, Arch Ophthalmol. 1949;39:808-812.

15. Hope-Simpson RE. The nature of herpes-zoster; A long term study and a new hypothesis. Proc Roy Soc Med. 1965;58:9-20.

16. Uchida Y, Kaneko M, Onishi Y. Ophthalmic herpes zoster without eruption. Acta XXIV International Congress of Ophthalmol. 1983; 876-879.

17. Burstein R, Yanitsky D, Goor-Aryeh I, et al. An association between migraine and cutaneous allodynia. Ann Neurol. 2000;47:614-624.

18. Hirayama K. Neurological semeiology, In: H Asai, editor. Facial pain. 2nd ed. Tokyo Bunkado press, 2006;323-332.

19. Arakawa I, Shimizu T. Correlation between allodynia in periorbital region and potential zoster virus in trigeminal or occipital nerves. Blackwell Publishing Ltd Cephalalgia. Abstracts of the 13 congress of the international headache society/13th IHC 2007; Stockholm, Sweden 28 June - 1 July 2007;27:575-759.

20. Nakamura M, Tanabe M, Yamada Y, et al. Zoster sine herpete with bilateral ocular involvement. Am J Ophthalmol. 2000;129:809-810.

21. Forghani B, Schmidt N, Dennis J. Antibody assays for varicella-zoster virus: comparison of enzyme immunoassay with neutralization, immune adherence hemaglutination, and complement fixation. Clinical Microbial. 1978;8:545-552.

22. Schaap GJ, Huisman J. Simultaneous rise in complement-fixing antibodies against herpesvirus hominis and varicella-zoster virus in patients with chickenpox and shingles. Arch ges Virusforsch. 1983;25: $52-57$. 
23. Abo T, Kawamura T. Immunomodulation by the autonomic nervous system: therapeutic approach for cancer, collagen diseases, and inflammatory bowel diseases. Ther Apheresis. 2002;6:348-357.
24. Abo T, Kawamura T, Kawamura H, et al. Relationship between diseases accompanied by tissue destruction and granulocytes with surface adrenergic receptors. Immunologic Res. 2007;37:201-210.

\section{Publish your work in this journal}

Clinical Ophthalmology is an international, peer-reviewed journal covering all subspecialties within ophthalmology. Key topics include: Optometry; Visual science; Pharmacology and drug therapy in eye diseases; Basic Sciences; Primary and Secondary eye care; Patient Safety and Quality of Care Improvements. This journal is indexed on
PubMed Central and CAS, and is the official journal of The Society of Clinical Ophthalmology (SCO). The manuscript management system is completely online and includes a very quick and fair peer-review system, which is all easy to use. Visit http://www.dovepress.com/ testimonials.php to read real quotes from published authors. 